\title{
Efectos mnemónicos maternos prenatales sobre el sexo psíquico humano. Insuficiencia del mecanismo inmunitario. Nueva propuesta desde la tolerancia-rechazo materno-fetal
}

\author{
Carlos Y Valenzuela \\ Prenatal maternal mnemonic effects \\ on the human neuro-psychic sex. \\ A new proposition from \\ fetus-maternal tolerance-rejection
}

In approximately $15 \%$ of homosexual men, their phenotype is associated to the fraternal birth order. Older biological brothers induce in their mothers anti-male factors (antibodies) that interfere the brain maleness development of younger fetuses. This effect is seldom seen in non-right-handed men and is not seen in women. The influence of older siblings is seen in their sex ratio (SR). In contradiction with previous hypothesis, significant heterogeneities of SR have been found among older siblings of males or females, right or non-right-handed and homo or heterosexual individuals. This can only be understood as if the findings found among homosexuals were part of a general mechanism of fetus-maternal tolerance-rejection processes of placental mammals. We found, in relation to $\mathrm{ABO}$ and Rh systems and sex, that embryos with genes different from those of their mothers, induced better pregnancies and maternal tolerance than embryos similar to their mothers. Assuming that homo or heterosexuality and right or nonright-handedness behave similar to $\mathrm{ABO}$ or $\mathrm{Rh}$ alleles, the author provides a speculative interpretation of these results. Homosexual women (lesbians) and especially if they are non-righthanded, are preceded by siblings with a high SR (maternal environment with anti-female or promale factors); then lesbianism or non-right-handedness may induce tolerance to be a woman in such anti-female environment. Non-right-handedness could induce tolerance for anti-male factors of mothers, thus preventing the production of gays in a pro-male maternal environment, but leading to the production of non-right-handed gays in anti-male maternal environments. Several new hypotheses and interpretations merge from this new proposition. Also, complete sexual orientation could be acquired after birth (Rev Méd Chile 2008; 136: 1552-8).

(Key words: Genotype; Phenotype; Sexual dysfunction, psychological; Homosexuality; Laterality)

Recibido el 3 de marzo, 2008. Aceptado el 11 de agosto, 2008.

Programa de Genética Humana, ICBM, Facultad de Medicina, Universidad de Chile

\footnotetext{
Correspondencia a: Dr. Carlos Y Valenzuela. Programa de Genética Humana. Instituto de Ciencias Biomédicas (ICBM), Facultad de Medicina, Universidad de Chile. Independencia 1027, Casilla 70061, Independencia, Santiago, Chile. Fax: (56-2) 7373158. Fonos: (56-2) 9786456-9786302. E mail: cvalenzu@med.uchile.cl
} 
$\mathrm{N}$ uestra proposición sobre el desarrollo del sexo psíquico y social humano ha sido sustentada con descubrimientos posteriores ${ }^{1,2}$. Estudios concluyentes indican que la orientación homosexual masculina es producida, en cerca de 15\% de los casos, por algún factor de memoria materna antimasculina generada por los hermanos biológicos varones mayores criados juntos o aparte $\mathrm{e}^{3-10}$. Esto debilita la interpretación psicoanalítica de la génesis de la orientación sexual (diamorfofilia según nuestra nomenclatura) ${ }^{1,2}$, ya que el ambiente postnatal influiría menos que el prenatal. Este efecto operaría sólo en homosexuales varones por factores inmunitarios ${ }^{3-10}$; dos propuestas contradictorias con los resultados publicados. Uno de los objetivos de este trabajo es mostrar tal contradicción. Esta relación se confirmó en transexuales ${ }^{11}$ en quienes la identidad y no la orientación sexual está afectada ${ }^{1,12,13}$. Afirmando la génesis neural prenatal del factor predisponente a la homosexualidad masculina se constató que este efecto se da en mucho menor grado en varones no-diestros ${ }^{12,14}$; a pesar que, los varones tienen mayor no-dextralidad (no usaremos dextrismo) que las mujeres y los homosexuales masculinos (gays) o femeninos (lesbianas) tienen más que los heterosexuales respectivos ${ }^{15,16}$. La interpretación postulada es dual: 1) la condición de no-dextralidad neutraliza hormonalmente al factor antimasculino materno; 2) la condición conjunta de no-dextralidad y homosexual por efecto materno prenatal sería letal o implicaría una condición mental que impide a estas personas acceder a los estudios ${ }^{14,16}$. Un segundo objetivo es proponer hipótesis alternativas a las presentadas, empleando sus mismos resultados. La influencia mnemónica materna puede examinarse por la proporción de los sexos [SR de sex ratio, (№ varones)/(№ varones + № de mujeres)] en los hermanos del índice. Los estudios muestran una gran desviación del SR poblacional en todas las categorías dadas por las condiciones de sexualidad o uso-manual ${ }^{7,17-19}$ (mi traducción de handedness); pero estos autores consideran las distorsiones sólo en gays y desestiman las encontradas en heterosexuales y lesbianas. El tercer objetivo es dar una visión integrada de todas las distorsiones asociadas a uso-manual, sexo, orientación sexual y SR en las fratrías e integrarla a la evolución de los placentados.
En 1980 descubrimos que los hermanos menores tendían a ser diferentes en sus genotipos $\mathrm{Rh}$ a sus hermanos previos lo que implicaba que las madres de un genotipo Rh, tenían menos progenie de ese genotipo en los embarazos sucesivos, y más de otros genotipos distintos a los de ellas aunque fueran incompatibles ${ }^{20-22}$. Encontramos también este efecto en el sistema ABO; las madres A tenían más hijos B y las madres B más hijos A que lo esperado ${ }^{23-25}$. El efecto Rh y el ABO fueron confirmados en muestras de Alemania, Australia, Inglaterra y Chile $\mathrm{e}^{23-25}$ y en todas ellas éste interactuaba con el $\mathrm{SR}^{23-26}$. La distorsión se encontró en el sistema HLA ${ }^{27}$ interactuando con el $\mathrm{SR}^{27-30}$. Nuestro descubrimiento tiene aún una sola interpretación ${ }^{25}$ : los cigotos, embriones o fetos que portan alelos (de algunos sistemas genéticos) desconocidos por su madre inducen mejor el embarazo y la tolerancia materna que aquellos que portan alelos iguales a los maternos; interpretación que llenó un vacío científico en la reproducción de los placentados ${ }^{31}$. La única forma que, en los placentados, sistemas alélicos, que inducen rechazo en las madres que no tienen el alelo de sus concepciones, puedan subsistir en la población, es asociarse a un mecanismo de inducción de tolerancia para esos alelos o para sistemas alélicos asociados. La tolerancia materna puede ser por camuflaje de los alelos del embrión, por falta de inducción del rechazo materno (tolerancia per se), por cambio molecular antigénico por interacción génica o por otros mecanismos ${ }^{23-31}$. Si las madres rechazan los embriones de fenotipos distintos a ellas, la población se hace homocigota y se esteriliza. Así se compatibiliza la permanencia de polimorfismos y variabilidad genética en los placentados. Se asocia a este mecanismo una distorsión del SR en relación a los pares madrehijo ${ }^{20-26}$, similar a la descrita para la homosexualidad y uso-manual. Las cifras confirman que los embriones y fetos masculinos inducen con mayor probabilidad un embarazo y tolerancia materna que los femeninos (SR sobre 0,5) en todos los órdenes de la fratría. La inducción de embarazo y tolerancia materna relacionada con los fenotipos Rh o ABO puede ser hacia sistemas genéticos ligados o interactivos evolutivamente con ellos ${ }^{31}$. Desde el embrión pasan células a la madre que garantizan su inmunización con antígenos embrionarios $^{32}$, fenómeno que no se corresponde con 
los inmunitarios conocidos; podría ser un sistema de tolerancia-rechazo materno inducido por acción molecular directa en el tránsito de los embriones.

Suponiendo que los genes que determinan la orientación sexual y (dextralidad) - (no-dextralidad) se comportan como el $\mathrm{Rh}$ y $\mathrm{ABO}$ para efectos materno-fetales, reinterpretaremos los datos de la literatura. Los genes involucrados en producir el fenotipo no-diestro podrían, en cierto contexto genómico, ocultar al sistema inmune de la madre, la masculinidad del cerebro del embrión por la inducción de tolerancia en estos fenotipos, por estar protegidos de la acción inmune de la madre, o por interacciones genéticas complejas que cambian molecularmente los fenotipos.

\section{MateRial y MÉTODO}

Tomamos dos estudios ${ }^{17,18}$ con individuos clasificados según sexo somático (masculino-femenino), orientación sexual (homo-hetero) y uso-manual [diestro-(no-diestro)] configurándose 8 categorías distintas y un tercer estudio ${ }^{19}$ realizado con varones exclusivamente. Los estudios describen el SR de los hermanos mayores ${ }^{17-19}$.

Racional. Planteamiento de los autores. Las madres que han tenido un embarazo con embrión masculino forman factores inmunitarios antimasculinos que en embarazos posteriores actúan sobre el cerebro embrionario o fetal masculino produciendo homosexualidad 8,33 . El antígeno masculino candidato es el HY que se desarrolla desde el estado de 8 células ${ }^{33-35}$. Mientras más hermanos varones anteriores tienen, más alta es la probabilidad de homosexualidad. Este planteamiento tiene debilidades inmediatas: i) el SR es superior a 0,5 (más varones que mujeres) en todos los órdenes de nacimiento ${ }^{36}$ (disminuye el SR con el orden en la fratría pero nunca bajo 0,5 ); ii) no todos los hermanos posteriores a un varón homosexual, nacidos de la misma madre son homosexuales ${ }^{11}$. Se han agregado hipótesis adicionales para mantener un efecto inmunitario materno modulado por acciones hipotéticas del mismo sistema inmune ${ }^{37}$; iii) se busca una regularidad causal de homosexualidad masculina (limitada al sistema inmune) con correlato lineal con ella y no se considera que la orientación sexual es un fenotipo complejo con heterogeneidad genética y que por interacciones no-lineales puede asociarse a diversos SR en distintas poblaciones que difieren en genotipo residual. Bajo este racional la metodología consiste en reunir muestras de muchas poblaciones, mezclar los datos y tratar de sacar algunas conclusiones regulares. Estos autores contrastan con un SR propuesto para la población humana con 106 varones por cada 100 mujeres, lo que resulta $S R=106 / 206=0,5145^{12-19}$.

Nuestro planteamiento. Los mecanismos involucrados no se limitan al sistema inmune ni a algún fenotipo de orientación sexual, uso-manual o valor de SR, sino que a la interacción genómica, evolutiva, compleja, materno-cigoto-embrión-feto de los animales placentados, que además de rechazar los embriones distintos a la madre por incompatibilidad inmune, deben mantener el polimorfismo genético de la especie para que no se extinga. Para que un cigoto o embrión induzca un embarazo y sea implantado debe poseer genes diferentes a su madre. Hay más varones que mujeres (al nacimiento), porque a igualdad genómica tienen los genes de masculinidad desconocidos por sus madres. Las madres no anidan o abortan más a sus embriones femeninos (similares a ellas); efecto distinto a lo inmunitario hasta hoy conocido. El tiempo de espera intergenésico apunta a la pérdida de embriones, similares a sus madres, en el primer mes de gestación ${ }^{21}$. Los embriones que poseen genes distintos a su madre desarrollan un mecanismo de tolerancia en sus madres, sea camuflando sus fenotipos (genes) distintos, sea paralizando al sistema inmune materno, lo que no es completo o para todas las diferencias, por lo que queda una parte de los aceptados sometida al rechazo inmunitario. Hay un equilibrio entre la inducción de embarazo y tolerancia y el rechazo. Como la pléyade genómica madre-hijo es muy compleja, cada pareja madre-hijo, muestra, población, etc., son irreducibles y no mezclables; el análisis debe hacerse con muestras separadas; sin excluir alguna regularidad interpoblacional. Los embriones femeninos también pueden inducir tolerancia-rechazo al poseer genes distintos a los de la madre. Además el genoma residual actúa impredeciblemente en la determinación de la orientación sexual y viabilidad fetal ${ }^{25,38,39}$. La relación entre orden fraterno 
masculino (fraternal birth order) y homosexualidad masculina es un aspecto de la genómica materno-fetal. El SR $=0,5145$ general sirve como primera aproximación, pero el SR de cualquier categoría debe estudiarse de acuerdo a su contexto genómico materno-fetal.

\section{Resultados}

Los datos detallados, análisis estadísticos e interpretación de los autores están en sus trabajos originales $^{3-19}$, aquí presentamos nuestra interpretación basada en: 1) resultados de los sistemas $\mathrm{ABO}, \mathrm{Rh}$ y HLA en las relaciones toleranciarechazo materno-fetales ${ }^{19-26,31,38,39}$ y 2) la nueva proposición sobre la definición y ontogenia del sexo psíquico y social ${ }^{1,2,12}$.

Datos y reinterpretación. La Tabla 1 presenta la proporción sexual (SR) en los hermanos mayores en dos muestras con 8 categorías fenotípicas y en dos trabajos con varones exclusivamente. La primera $^{17}$, tercera ${ }^{19}$ y cuarta $^{19}$ muestras contienen fenotipos metodológicamente equivalentes, la segunda ${ }^{18}$ fue obtenida por una encuesta masiva de la BBC (British Broadcasting Corporation) con categorías fenotípicas distintas (homosexuales y bisexuales juntos, no estrictamente comparable). Nosotros no fusionamos muestras y buscamos hetero y homogeneidades cuali-cuantitativas. La única categoría asimilable en los cuatro estudios es la de los heterosexuales masculinos. Las significaciones estadísticas corresponden a la prueba $\chi^{2} \mathrm{k}$ ( $\mathrm{k}$ grados de libertad) para heterogeneidad de una proporción (SR). Hay dos resultados notables: 1) la enorme heterogeneidad de SR en hermanos mayores de los varones heterosexuales diestros $\chi_{3}^{2}=11,5(\mathrm{P}=0,009)$ y no-diestros $\chi_{3}^{2}=17,9(\mathrm{P}$ $=0,0005)$, que contrasta con la homogeneidad encontrada en las mujeres de los estudios $1^{\circ}$ y $2^{\circ}$, que para las diestras fue $\chi^{2}{ }_{1}=1,6(\mathrm{P}=0,209)$ y para las no-diestras fue $\left.\chi^{2}{ }_{1}=2,69(\mathrm{P}=0,101) ; 2\right) \mathrm{El}$ elevado SR encontrado en las mujeres homosexuales (lesbianas) no-diestras que en las dos muestras comparables fue el más alto de todas las categorías de estudio $(0,65$ y 0,541 , respectivamente).

$\mathrm{Al}$ incorporar a las mujeres bisexuales en el estudio $2^{\circ}$ como lesbianas, habría un descenso del SR en las lesbianas no-diestras (de 0,65 a 0,541). Estudiamos si había diferencia significativa entre ambos y así resultó $\chi^{2}{ }_{1}=5,71(\mathrm{P}=0,016)$, pero no hubo significación en las lesbianas diestras de ambos estudios $^{17,18}\left(\chi_{1}^{2}=0,239, \mathrm{P}=0,625\right)$ aunque ambas tuvieron mayor SR que la población gene-

Tabla 1. Proporción sexual (SR) en hermanos mayores

\begin{tabular}{|c|c|c|c|c|c|c|c|c|}
\hline \multirow[t]{2}{*}{ Categoría } & \multicolumn{2}{|c|}{ 10 Estudio } & \multicolumn{2}{|c|}{ 20 Estudio } & \multicolumn{2}{|c|}{ 30 Estudio } & \multicolumn{2}{|c|}{ 4ํ Estudio } \\
\hline & $\mathbf{N}$ & SR & $\mathbf{N}$ & SR & $\mathbf{N}$ & SR & $\mathbf{N}$ & SR \\
\hline \multicolumn{9}{|c|}{ Varón heterosexual } \\
\hline Diestro & 589 & 0,553 & 61.899 & 0,502 & 2.213 & 0,489 & 3.331 & 0,519 \\
\hline No-diestro & 86 & 0,465 & 9.049 & 0,498 & 324 & 0,608 & 405 & 0,541 \\
\hline \multicolumn{9}{|c|}{ Varón homosexual } \\
\hline Diestro & 686 & 0,555 & 7.311 & 0,511 & 1.241 & 0,565 & 837 & 0,560 \\
\hline No-Diestro & 190 & 0,453 & 1.311 & 0,498 & 174 & 0,552 & 85 & 0,447 \\
\hline \multicolumn{9}{|c|}{ Mujer heterosexual } \\
\hline Diestro & 656 & 0,502 & 52.479 & 0,526 & & & & \\
\hline No-Diestro & 133 & 0,444 & 6.317 & 0,515 & & & & \\
\hline \multicolumn{9}{|c|}{ Mujer homosexual } \\
\hline Diestro & 765 & 0,531 & 4.902 & 0,540 & & & & \\
\hline No diestro & 143 & 0,650 & 669 & 0,541 & & & & \\
\hline
\end{tabular}


ral $(S R=0,531, P=0,20$ y $S R=0,540, P=0,0004$, respectivamente). El resultado más significativo, es la alta proporción de varones en los hermanos mayores de las lesbianas no-diestras. Es notable la homogeneidad del alto SR (cerca de 0,56) en varones homosexuales (gays) diestros en las muestras comparables (estudios $1^{\circ}, 3^{\circ}$ y $4^{\circ}, \chi^{2}{ }_{2}$ $=0,16 \mathrm{P}=0,921)$ que contrasta con la casi significativa heterogeneidad de los no-diestros $\left(\chi_{2}^{2}=4,34\right.$, $\mathrm{P}=0,114)$.

\section{DisCUSIÓN}

Uno de los autores ${ }^{19}$ fusionó las muestras ( $1^{\circ}$, $3^{\circ}$ y $4^{\circ}$ ) obteniendo promedios de SR que no representan a ninguna y concluye sesgadamente sólo sobre los gays diestros, considerando los otros resultados como difíciles de interpretar. Los datos contradicen concluyentemente las hipótesis de los autores: i) la distorsión del SR afecta sólo a gays; pero la distorsión mayor afecta a las lesbianas y a varones heterosexuales; ii) los gays diestros serían producidos por factores antimasculinos; pero los gays diestros se dan en fratrías con altos SR por lo que las madres tendrían, contrariamente, factores promasculinos o antifemeninos; iii) se trata de un fenómeno inmunitario exclusivo, pero la distorsión del SR aparece en todas las categorías de hermanos, hecho que muestra a factores no inmunes jugando un rol principal. Desde nuestro racional, los resultados se ajustan a lo esperado y aparecen muchas interpretaciones, cada una de ellas con sus diseños de investigación. Las distorsiones del SR en los hermanos mayores, respecto al SR promedio o al SR de cada muestra, ocurren en todas las categorías fenotípicas de orientación sexual y uso-manual y no se confinan a los gays exclusivamente. La distorsión más grande de SR es la de las lesbianas no-diestras, resultado que los autores no consideran ${ }^{17}$, a pesar de que también las lesbianas diestras presentan SR más alto que el de la población general. Las familias con alto SR tienen una gran probabilidad de tener hijas lesbianas no-diestras o gays diestros, hecho corroborado por un estudio que no dividía por uso-manual ${ }^{40}$. Las madres de estas fratrías tienen un factor promasculino o antifemenino (alto SR) fuerte y los embriones femeninos en este ambiente adverso se favorecerían si difirieren de sus madres teniendo genes para ser lesbianas nodiestras (¿asimilación al fenotipo masculino?). Los gays diestros que también se dan en un ambiente materno promasculino o antifemenino no tienen dificultad para nacer varones en un ambiente promasculino, pero esto no es suficiente para evadir el factor antimasculino específico cerebral; si son no-diestros podrían evadir el factor antimasculino cerebral de sus madres. Mientras este trabajo se escribía apareció uno que plantea la posibilidad de inducción de tolerancia o resistencia de los embriones a los factores antimasculinos de las madres, como aquí se postulan ${ }^{41}$. Entre el cigoto o embrión y su madre podría haber una relación metabólica incompatible o una incapacidad de ser aceptado en la implantación, diferencias captadas en la conversación molecular entre el cigoto o embrión con su madre al pasar por el oviducto $^{42,43}$.

Nuestra discrepancia con los autores respecto a los factores materno-fetales no es la única. Ellos suponen que la orientación sexual es determinada prenatalmente ${ }^{3-11}$. Esto es insostenible. El hecho que se mantenga el SR superior a 0,5 en todos los hijos, sin importar el orden de nacimiento ${ }^{38,40,44}$ y sea similar en los hermanos mayores y menores al índice $40,41,44$, el que los hijos posteriores a un homosexual no sean todos homosexuales, el que este factor alcance a sólo $15 \%$ de los homosexuales, puede explicarse por una fijación postnatal de la orientación sexual nuclear (diamorfofilia ${ }^{1,2,12}$ ). La homogeneidad del SR superior a 0,5 contrasta con su enorme heterogeneidad cuando se clasifican las fratrías, binomios madre-hijo o padre-hijo por sus fenotipos (sexuales, uso-manual, ABO, Rh, etc.) donde se encuentran SR entre 0,0 y $1,0^{25,26,38,39}$. Los factores profemeninos (antimasculinos) o promasculinos (antifemeninos) determinarían una propensión a ser homosexual en un ambiente postnatal formador de diamorfofilia común. Desde la fenomenología de la diamorfofilia, nos enamoramos, vinculamos o relacionamos con una figura humana que no está en el genoma, ni en hormonas, ni en redes neurales, sino que en nuestros padres, madres y personas del ambiente postnatal. La diamorfofilia es un fenotipo complejo dado por factores prenatales condicionantes de la ontogenia psiconeural de la identidad sexual ${ }^{1,2,12}$, que se completa postnatalmente, probablemente por imprinting en un ambiente sano de vincula- 
ciones y valoraciones de lo-masculino y lo-femenino ${ }^{1,2,12}$. Este fenotipo tendría un factor neural básico, una capacidad de configurar formas sexuales, una capacidad de vinculación sexual, afectiva, emotiva y moral y un período crítico para que esto ocurriera. En estas instancias, pueden producirse alteraciones que lleven a una diamorfofilia inconsistente con los otros fenotipos sexuales, en diferentes períodos de la ontogenia ${ }^{1,2,12}$. Hay evidencias que el dimorfismo sexual neural

\section{REFERENCIAS}

1. Valenzuela CY. Fenotipos sexuales psicosociales. Una proposición y su ontogenia. Rev Méd Chile 1993; 121: 693-8.

2. Gaete Je, Valenzuela CY. Bases biológicas de la homosexualidad. En : Zegers B, Larraín ME, Bustamante F. Eds. Homosexualidad. Santiago: Mediterráneo, 2007; 99-134.

3. Blanchard R, Bogaert AF. Homosexuality in men and number of older brothers. Am J Psychiatry 1996; 153: 27-31.

4. Zucker KJ, Green R, Coates S, Zuger B, Cohen-Kettenis PT, ZECCA GM ET AL. Sibling sex ratio of boys with gender identity disorder. J Child Psico Psychiatry 1997; 38: 543-51.

5. BLANCHARD R. Fraternal birh order and the maternal immune hipothesis of male homosexuality. Horm Behav 2001; 40: 105-14.

6. Cantor JM, Blanchard R, Paterson ad, Bogaert aF. How many gay men owe heir sexual orientation to fraternal birth order. Arch Sex Behav 2002; 31: 63-71.

7. James J. The cause(s) of the fraternal birth order effect in male homosexuality. J Biosoc Sci 2004; 36: 51-9.

8. Bogaert AF. Sibling sex ratio and sexual orientation in men and women: new test in two national probability samples. Arch Sex Behav 2005; 34: 111-6.

9. BOgaert AF. Biological versus nonbiological older brothers and men's sexual orientation. Proc Natl Acad Sci (USA) 2006; 103: 10771-4.

10. Puts DA, Jordan CL, Breedlove SM. O brothers, where art thou? The fraternal birth-order effect on male sexual orientation. Proc Natl Acad Sci (USA) 2006; 103: 10531-2.

11. GREEN R. Birth order and ratio of brothers to sisters in transsexuals. Psychol Med 2000; 30: 789-95.

12. Valenzuela CY. La Homosexualidad ¿es una patología? Respuesta desde la biología evolutiva. Rev Psiquiat Clin (Santiago, Chile) 2006; 43: 27-38.

13. BOgaert AF. Gender role/identity and sibling sex ratio in homosexual men. J Sex Marital Ther 2005; 31: 217-27. se completa postnatalmente como lo hemos demostrado para núcleos del rafe ${ }^{45,46}$.

Nota: una versión extendida de estas ideas preliminares, con datos de los hermanos anteriores y posteriores al índice, enmarcada en la teoría general de la producción de machos y hembras en los placentados y de las relaciones materno-fetales está en revisión ${ }^{47}$.

14. Blanchard R, Cantor JM, Bogaert aF, Breedlove SM, ELLIS L. Interaction of fraternal birth order and handedness in the development of male homosexuality. Horm Behav 2006; 49: 405-14.

15. Lippa RA. Handedness, sexual orientation, and gender-ralated personality traits in men and women. Arch Sex Behav 2003; 32: 103-14.

16. BOgaert AF. Handedness in homosexual and heterosexual men in the Kinsey interview data. Arch Sex Behav 1996; 25: 373-8.

17. Blanchard R, Lippa RA. The sex ratio of older siblings in non-right-handed homosexual men. Arch Sex Behav 2006 DOI 10.1007/s10508-006-9107-6.

18. Blanchard R, Lippa RA. Birth order, sibling sex ratio, handedness, and sexual orientation of male and female participants in a BBC Internet Research Project. Arch Sex Behav 2007; 36: 163-76.

19. Blanchard R. 2006. Sex ratio of older siblings in heterosexual and homosexual right-handed and non-right-handed men. Arch Sex Behav DOI 10.1007/s10508-006-9119-2.

20. Valenzuela CY, Avendaño A, Harb Z, Acuña M. Análisis del grupo sanguíneo $\mathrm{Rh}$ en fratrías. ¿Un nuevo sistema de histocompatibilidad en el hombre? Rev Chil Pediatr 1981; 52: 125-32.

21. Valenzuela CY, Harb Z, Avendaño A, Acuña MP. A large sib-sib discordance for the Rh blood system. A possible new feto-maternal compatibility system. Am J Hum Genet 1982; 34: 576-89.

22. ValenZuela CY, Harb Z. A mother-child segregation distortion for the Rh system. New evidence for another compatibility system associated with Rh. Am J Hum Genet 1982; 34: 925-36.

23. Valenzuela CY. Confirmación de las distorsiones de los sistemas ABO y Rh y de la proporción sexual en recién nacidos. Rev Méd Chile 1985; 113: 1175-87.

24. Valenzuela CY, Lyng C, Armanet L, Islas E, Harb Z, Acuña MP. Anomalías segregacionales del sistema sanguíneo ABO asociadas al sistema Rh. Interpretación del efecto SAN. Rev Méd Chile 1984; 112: 213-7.

25. Valenzuela CY, Walton R. Selective interactions 
among Rh, $\mathrm{ABO}$, and sex ratio of newborns. Hum Genet 1985; 71: 53-7.

26. Valenzuela CY. Interacciones $\mathrm{ABO}, \mathrm{Rh}$ y sexo en recién nacidos. Rev Méd Chile 1985; 113: 472-3.

27. Ober Cl, Martin AO, Simpson Jl, Hauck WW, Amos DB, Kostyu DD et al. Shared HLA antigens and reproductive performance among Hutterites. Am J Hum Genet 1983; 35: 994-1004.

28. Astolfi P, Martinetti M, Gigli-Berzolari F, Cuccia M. The effect of parental and maternal-fetal histocompatibility at MHC on sex ratio in offspring. Tissue Antigens 1990; 35: 172-7.

29. Astolfi P, Cuccia M, Caruso C, Favoino B, Fazzari M, Mantovani V ET al. Sharing at the major histocompatibility complex affects the secondary sex ratio in differing ways. Hum Hered 1996; 46: 155-65.

30. Beydoun H, Saftlas AF. Association of human leucocyte antigen sharing with recurrent spontaneous abortion. Tissue Antigens 2005; 65: 123-35.

31. Valenzuela CY. Polimorfismos genéticos y compatibilidad materno-fetal. Dos problemas biológicos, una solucón evolutiva. Ann Univ Chile 1987; 5 serie, 14: 151-2.

32. Johnson KL, Stroh H, Khosrotehrani K, Bianchi DW. Spot counting to locate fetal cells in maternal blood and tissue: A comparison of manual and automated microscopy. Microsc Res Tech 2007; 70: 585-8.

33. Blanchard R, Klassen P. H-Y antigen and homosexuality in men. J Theor Biol 1997; 185: 373-8.

34. White KL, Anderson GB, Bondurant RH. Expression of a male-specific factor on various stages of preimplantation bovine embryos. Biol Reprod 1987; 37: 867-73.

35. Shelton JA, Goldberg EH. Male-restricted expression of $\mathrm{H}-\mathrm{Y}$ antigen on preimplantation mouse embryos. Transplantation 1984; 37: 7-8.
36. Biggar RJ, Wohlfahrt J, Westergand T, Melbye M. Sex ratios, family size, and birth order. Am J Epidem 1999; 150: 957-62.

37. BlanChaRD R. Quantitative and theoretical analyses of the relation between older brothers and homosexuality in men. J Theor Biol 2004; 230: 173-87.

38. Valenzuela CY. Are there true ABO and Rh segregation distortions? Braz J Genet 1996; 19: 343-9.

39. Kumari JR, Srikumari CR, Valenzuela CY. ABO segregation distortion in Visakhapatnam, India. Anthrop Ans 1992; 50: 307-14.

40. Bogaert AF. Sibling sex ratio and sexual orientation in men and women: new tests in two national probability samples. Arch Sex Behav 2005; 34: 111-6.

41. Blanchard R. Review and theory of handedness, birth order, and homosexuality in men. Laterality 2008; 13: 51-70.

42. Barnea ER. Embryo maternal dialogue: From pregnancy recognition to proliferation control. Early Pregnancy 2001; 5: 65-6.

43. Krussel JS, Bielfeld P, Polan ML, Simon C. Regulation of embryonic implantation. Eur J Obstet Gynecol Reprod Biol 2003; 110 Suppl 1: S2-S9.

44. Mathews TJ, Hamilton BE. Trend analysis of the sex ratio at birth in the United States. Natl Vital Stat Rep (USA) 2005; 53: 1-17.

45. Cordero me, Valenzuela Cy, Torres R, Rodríguez A. Sexual dimorphism in number and proportion of neurons in the human median raphe nucleus. Dev Brain Res 2000; 124: 43-52.

46. Cordero me, Rodríguez A, Torres R, Valenzuela Cy. Human Raphe Magnus Nucleus: a morphometric Golgi-Cox study with emphasis on sex differences. Dev Brain Res 2001; 131: 85-92.

47. Valenzuela CY. Sexual orientation, handedness, sex ratio and fetomaternal tolerance-rejection. Laterality (in revision). 